\title{
Neurodegeneration with Brain Iron Accumulation (NBIA)- Hallervorden-Spatz Syndrome; a case report
}

\author{
Kathriarachchi Samudra ${ }^{1}$, Sabapathippillai Saumyarika ${ }^{2}$, Gunawardena Dulmini ${ }^{3}$, Riffsy MTM ${ }^{3}$ \\ ${ }^{1}$ Faculty of Medical Sciences, University of Sri Jayewardenepura, Nugegoda, ${ }^{2}$ Teaching Hospital \\ Karapitiya, ${ }^{3}$ Colombo South Teaching Hospital, Kalubowille, Sri Lanka
}

Correspondence to: Dr. Saumyarika Sabapathippillai (saumy1@yahoo.com)

\section{Introduction}

NBIA is a group of rare autosomal recessively inherited, progressive extrapyramidal disorders characterised by typical clinical and radiological features with evidence of focal iron accumulation mainly in the basal ganglia $(1,2)$.

\section{Case Report}

We report a 32-year old male who had initially presented with a history of impulsiveness and violent outbursts and social withdrawal five years ago. At that time there were no neurological symptoms. Exact diagnosis was not established and a behavioural programme was commenced. He was started on low dose of risperidone. However, he defaulted treatment.

He presented in September 2010 with difficulty in walking for about 18 months which had become worse during the last three months. On direct inquiry he reported his predicament started as a mild tremor of hands and lips 4 years ago. Three weeks prior to this presentation, his speech had become more slurred. He never had dysphagia, visual disturbance, seizures or headache. There was no family history of similar illnesses. Apart from above his medical history was unremarkable. He functioned as a mechanic but had not been able to do his job for the last 18 months.

His general examination was normal. He had dysarthritic speech, spastic rigid limbs with grade 4 power, and bilateral hyper-reflexia. Cranial nerves and eye movements were normal. His gait was broad based with marked unsteadiness. Funduscopy was normal. On slit lamp examination, no KeischerFleischer rings were found. He was depressed with impaired cognition. His mini mental score was 17/30.There was no thought disorder.
His full blood count, ESR, renal and liver functions were normal. Serum ceruloplasmin and copper and iron studies were within normal limits. T2 weighted magnetic resonance imaging(MRI) showed the hall mark sign of NBIA 'the eye of tiger' sign, an area of hyperintensity in the antero medial globuspallidum, surrounded by hypo-intensity in the rest of the pallidal region and substantianigrareticulata bilaterally (Figure). Due to financial restraints genetic analysis was not done.

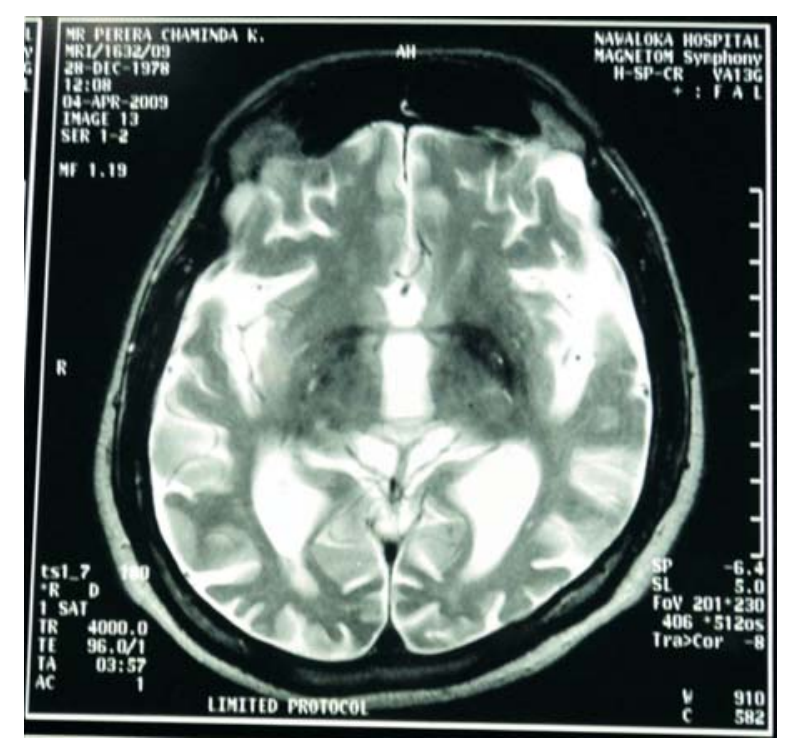

Figure: MRI Brain showing 'the eye of tiger' sign

\section{Discussion}

Initially NBIA was named by Hallervorden and Spatz, as they first described it in five sisters of 12 siblings in 1922. The genetic basis of the disease was found in 2001 and is considered to be due to a mutation in pantothenate kinase gene (PANK2) in chromosome 20. Typical, atypical and intermediate forms of this condition exist $(1,2)$. 
Typical form presents within the first decade with rapidly progressive extrapyramidal syndrome, truncal dystonia, chorea, dysarthria, pyramidal signs, gait disturbance, cognitive impairment and delay in milestones, often with pigmentary retinopathy. Atypical form begins in the second decade with slow progression. Intermediate form is characterised by either early onset and slow progression or late onset with rapid progression. Our patient falls into the atypical group.

PANK2 mutation causes error in the biosynthesis of Co-enzyme A, resulting in accumulation of cysteine $(1,3)$. This causes tissue damage and oedema in certain brain regions (globuspallidum, substantianigra) producing central hyperintensity in MRI. Furthermore, cysteine chelates iron producing the peripheral hypointensity as described above $(1,3)$. Systemic iron metabolism, however, is normal (1).

Diagnostic criteria: Obligatory features are, onset within first three decades, progressive signs and symptoms, extra-pyramidal dysfunction, the 'eye of tiger' sign on MRI (T2 weighted) brain; while corroborative features include corticospinal tract involvement, progressive intellectual impairment, retinitis pigmentosa / optic atrophy and positive family history $(1,4)$. For diagnosis all obligatory and two corroborative features are needed (4). Our patient fulfilled these. He had no exclusion features such as abnormal copper or caeruloplasmin levels, family history of Huntington's chorea, predominant epileptic symptoms, severe visual impairment, caudate atrophy and absence of extra-pyramidal features (4).

All patients with this PANK2 mutations had the specific pattern on MRI brain T2 weighted images, known as 'the eye of tiger' sign. ${ }^{3}$ PANK2 gene mutations were seen in all classic cases and $1 / 3$ of cases of atypical form. ${ }^{3}$ Although there is high variability in presentation, brain MRI findings will often lead clinicians to this diagnosis (3).
Management of NBIA mainly aims toward symptomatic treatment (levodopa, carbidopa, bromocriptine, deep brain stimulation) but none have proved to be effective $(1,4)$. Patients with typical form loose their ability to ambulate 10-15 years after the onset of the disease and die by the age of 35 years. The atypical patients loose their independent ambulation 15-40 years after onset (1).

Levodopa was commenced and practical measures (speech and physiotherapy) were initiated in our patient. Family was informed about the prognosis.

This report highlights the phenotypic diversity of NBIA and emphasizes that an undiagnosed patient might present at different settings. Awareness of the syndrome is important in making an early diagnosis. To the best of our knowledge no case of NBIA has been reported in the medical literature in Sri Lanka.

\section{References}

1. Allison G, Hayflicks SJ. Neurodegeneration with Brain Iron Accumulation. Folia Neuropathol, 2005; 43(4): 286-296.

2. Sachin S, Goyal V, Singh S, Shukla G, Sharma MC,Geikwel S, Behari M. Clinical Spectrum of HallervordenSpatz Syndrome in India. J Neurol Sci, 2009; 16(2): 253-258.

3. Hayflick SJ, Hartman M, Coryell J, Gitschier J, Rowleg H. Brain MRI in Neurodegeneration with Brain Iron Accumulation with or without PANK 2 mutation. Am JNeuroradiol, 2006; 27(6): 1230-1233.

4. Kenneth F, Swaiman. HallervordenSpatz Syndrome and Brain Iron Metabolism. ArchNeurol, 1991; 48: 1285-1293. 